\title{
Optimal systolic blood pressure target, time to intensification, and time to follow-up in treatment of hypertension: population based retrospective cohort study
}

\author{
Wenxin Xu, Saveli I Goldberg, ${ }^{2}$ Maria Shubina, ${ }^{3}$ Alexander Turchin ${ }^{3}$
}

Department of Medicine, Beth Israel Deaconess Medical

Center, Boston, MA 02115, USA

${ }^{2}$ Department of Radiation

Oncology, Massachusetts

General Hospital, Boston,

MA 02114, USA

${ }^{3}$ Division of Endocrinology,

Brigham and Women's Hospital,

221 Longwood Avenue, Boston,

MA 02115, USA

Correspondence to:

A Turchin aturchin@partners.org

Additional material is published online only. To view please visit the journal online (http:// dx.doi.org/10.1136/BMJ.h158)

Cite this as: $B M J$ 2015;350:h158 doi: 10.1136/bmj.h158

Accepted: 9 December 2014

\section{ABSTRACT}

OBJECTIVES

To investigate the optimal systolic blood pressure goal above which new antihypertensive medications should be added or doses of existing medications increased ("systolic intensification threshold") and to determine the relation between delays in medication intensification and follow-up and the risk of cardiovascular events or death.

DESIGN

Retrospective cohort study.

SETTING

Primary care practices in the United Kingdom, 1986-2010.

\section{PARTICIPANTS}

88756 adults with hypertension from The Health Improvement Network nationwide primary care research database.

\section{MAIN OUTCOME MEASURES}

Rates of acute cardiovascular events or death from any cause for patients with different hypertension treatment strategies (defined by systolic intensification threshold, time to intensification, and time to follow-up over the course of a 10 year treatment strategy assessment period) after adjustment for age, sex, smoking status, socioeconomic deprivation, history of diabetes, cardiovascular disease or chronic kidney disease, Charlson comorbidity index, body mass index, medication possession ratio, and baseline blood pressure.

\section{WHAT IS ALREADY KNOWN ON THIS TOPIC}

In the routine management of hypertension, the optimal systolic blood pressure goal above which new antihypertensive medications should be added or existing doses increased is not well understood

The optimal time interval between the measurement of a blood pressure above goal and intensification of the antihypertensive regimen and the optimal time to reassess blood pressure after medication intensification are not known

\section{WHAT THIS STUDY ADDS}

Systolic intensification thresholds higher than $150 \mathrm{~mm} \mathrm{Hg}$ were associated with increased risk of subsequent acute cardiovascular events or death

Delays of greater than 1.4 months before medication intensification after systolic blood pressure elevation and delays of greater than 2.7 months before blood pressure follow-up after antihypertensive medication intensification were associated with increased risk

These findings support the importance of avoiding delays in medical management and follow-up in the treatment of patients with hypertension

\section{RESULTS}

During a median follow-up of 37.4 months after the treatment strategy assessment period, 9985 (11.3\%) participants had an acute cardiovascular event or died. No difference in risk of the outcome was seen between systolic intensification thresholds of 130-150 mm Hg, whereas systolic intensification thresholds greater than $150 \mathrm{~mm} \mathrm{Hg}$ were associated with progressively greater risk (hazard ratio 1.21, 95\% confidence interval 1.13 to $1.30 ; \mathrm{P}<0.001$ for intensification threshold of $160 \mathrm{~mm} \mathrm{Hg}$ ). Outcome risk increased progressively from the lowest (0-1.4 months) to the highest fifth of time to medication intensification (hazard ratio 1.12, 1.05 to 1.20; $\mathrm{P}=0.009$ for intensification between 1.4 and 4.7 months after detection of elevated blood pressure). The highest fifth of time to follow-up (> 2.7 months) was also associated with increased outcome risk (hazard ratio $1.18,1.11$ to $1.25 ; \mathrm{P}<0.001$ ).

\section{CONCLUSIONS}

Systolic intensification thresholds higher than $150 \mathrm{~mm} \mathrm{Hg}$, delays of greater than 1.4 months before medication intensification after systolic blood pressure elevation, and delays of greater than 2.7 months before blood pressure follow-up after antihypertensive medication intensification were associated with increased risk of an acute cardiovascular event or death. These findings support the importance of timely medical management and follow-up in the treatment of patients with hypertension.

\section{Introduction}

Hypertension is the single most common risk factor for both cardiovascular and overall disease burden and mortality worldwide, and the medical treatment of hypertension mitigates this risk. ${ }^{1-4}$ Management of hypertension is also among the most common reasons for ambulatory visits to physicians' clinics among non-pregnant adults worldwide. ${ }^{256}$ However, many key aspects of optimal medical management for hypertension remain unclear.

The evidence that medically treating patients with stage 1 (140-159 mm Hg) systolic hypertension improves outcomes is limited, and current guidelines differ substantially in their recommendations for management. ${ }^{6-9}$ In addition, for all patients little evidence is available to guide the optimal time interval between measurement of elevated blood pressure and addition or dose escalation of antihypertensive medications ("medication intensification") or between medication intensification and follow-up measurement of blood pressure. 
Routine clinical practice differs from clinical trials in that substantial delays may exist between the observation of an elevated blood pressure and medication intensification, ${ }^{1011}$ or between medication intensification and follow-up measurement of blood pressure, but the effect of such delays on patients' outcomes is not understood.

We therefore did a retrospective cohort study to investigate the systolic intensification threshold, time to intensification, and time to follow-up that are associated with the lowest risk of cardiovascular events or death.

\section{Methods}

Data source

The Health Improvement Network is an electronic medical record database containing data on encounters with patients collected from primary care practices throughout the United Kingdom that choose to submit their electronic records for research purposes. Patients' and practices' characteristics within The Health Improvement Network database are representative of those in the general UK primary care population. ${ }^{12}$ General practitioners are required to document encounters with patients through a process that is subject to audit, and the accuracy of patients' records is linked with compensation via the UK quality and outcomes framework. Patients' mortality and dates of death in The Health Improvement Network are assessed using information that is forwarded to the patient's general practitioner on administration of the death certificate. The accuracy of diagnosis and death records in the database have been previously validated..$^{13} 14$

\section{Study cohort}

We included adults registered in primary care practices in The Health Improvement Network database between 1986 and 2010. We included all patients who were aged 18 years or older, had at least one diagnosis code related to hypertension (supplementary table A), had at least one blood pressure measurement and at least one antihypertensive medication started or intensified, had at least 10 years of continuous data (to allow adequate time to assess treatment strategy), and had at least one set of height and weight data. To permit adjustment for socioeconomic deprivation, we excluded patients with missing postal codes. We also excluded patients with body mass index less than 15 or greater than 100 to minimize the effect of implausible values.

\section{Study measurements}

Patients' baseline characteristics and pre-existing medical conditions were assessed during the run-in period. ${ }^{15}$ The run-in period began on the clinic registration date and ended on the later date of 12 months after clinic registration or the first documentation of a hypertension related diagnosis code or systolic blood pressure of $130 \mathrm{~mm} \mathrm{Hg}$ or above.

We defined a treatment strategy assessment period for each patient, which consisted of the first 10 years after the end of the run-in period. During the treatment strategy assessment period, we defined the minimum intensification threshold as the lowest systolic blood pressure at which antihypertensive medication intensification occurred (out of all known intensification events), rounded down to the nearest $10 \mathrm{~mm} \mathrm{Hg}$. For instance, for a patient who had antihypertensive medication additions or dose increases at blood pressures of 167, 145, and $155 \mathrm{~mm} \mathrm{Hg}$, we would assume that the physician was targeting a blood pressure goal of $140 \mathrm{~mm} \mathrm{Hg}$. This approach allowed us to represent the patient's treatment as a single summary, as is the standard approach in cohort studies. ${ }^{16}$ We defined antihypertensive medication intensifications as the start of a new antihypertensive medication (complete list in supplementary table B) or an increase in the daily dose of an existing antihypertensive medication, on a date on which blood pressure was measured. ${ }^{17}$ Medication adjustments made when systolic blood pressure was already lower than $130 \mathrm{~mm} \mathrm{Hg}$ are unlikely to represent antihypertensive medication intensifications, so we excluded them from the analysis to minimize confounding from secondary indications for these medications (angiotensin converting enzyme inhibitors prescribed for patients with diabetes and renal failure, for instance).

We defined time to intensification as the mean length of unintensified hypertensive periods; each hypertensive period started on the day when systolic blood pressure was first measured to be above the minimum intensification threshold and ended on the first subsequent day when medications were intensified or when the unintensified period was censored (for example, systolic blood pressure fell below the threshold). Transient blood pressure elevations, defined by a single elevated blood pressure measurement above the intensification threshold that fell below the threshold at the next blood pressure reading in the absence of medication intensification, were excluded from the analysis. We defined time to follow-up as the mean time between each medication intensification and the next visit at which blood pressure was recorded.

We defined time to outcome as the time elapsed between the end of the treatment strategy assessment period and the composite outcome, which we defined as a first acute cardiovascular event (myocardial infarction, cerebrovascular accident, acute congestive heart failure episode, or peripheral vascular disease) or death (supplementary table C).

We calculated age at the conclusion of the run-in period. We calculated body mass index by using the first set of height and weight data available for each patient. We defined smoking history as past or current tobacco use and chronic kidney disease as the presence of a diagnosis code associated with chronic kidney disease (except stage 1 or 2 chronic kidney disease) or an estimated glomerular filtration rate less than $60 \mathrm{ml} / \mathrm{min} / 1.73 \mathrm{~m}^{2}$ (supplementary table C). ${ }^{18}$ We calculated a medication possession ratio (as a proxy for compliance) for each patient as a weighted average of the number of days' supply actually prescribed for each medication, divided by the total period of time over 
which that medication was prescribed. ${ }^{19}$ We calculated the Charlson comorbidity index from the Read code list by using a previously described and validated method, while excluding conditions individually included in the multivariable analysis. ${ }^{20}$

We estimated socioeconomic status by using the Townsend socioeconomic deprivation score, which is calculated using economic and demographic data based on the patient's postal code..$^{21}$ Patients who had multiple addresses during the follow-up period had their Townsend deprivation score calculated as a weighted average based on time spent living at each address.

\section{Statistical analysis}

We produced summary statistics by using frequencies and proportions for categorical variables and means, standard deviations, medians, and ranges for continuous variables. We used a Cox proportional hazards regression model to compare event-free survival for patients with various treatment strategies as defined by systolic intensification threshold, time to intensification, and time to follow-up..$^{22}$ The analysis was adjusted for the entry age category $(<60,60-74, \geq 75), 202324$ as well as for sex, smoking status, Townsend score, previous history of diabetes/cardiovascular disease/chronic kidney disease, Charlson comorbidity index, body mass index, medication possession ratio, and the mean difference by which systolic blood pressure exceeded the minimum intensification threshold at the beginning of each hypertensive period. ${ }^{25}$ We used multiple imputation in the sensitivity analysis that included patients with missing demographic or body mass index information.

We anticipated that treatment target, time to intensification, and time to follow-up may have a non-linear relation to risk of an event, with an optimal range of values and increased hazards outside this range. On the other hand, thresholds defined by fifths of each variable are somewhat arbitrary, with the boundaries determined by the behavior of the physicians in our particular sample. To provide an alternative view of the relation between systolic intensification threshold, time to intensification, time to follow-up, and event risk, we constructed Cox regression models that included systolic intensification threshold, time to intensification, and time to follow-up as natural cubic splines to account for a continuous non-linear functional dependence between these treatment parameters and the log hazard rate. Spline knots were placed at the 5th, 25th, 75th, and 95th centiles of the overall distribution of each of the three variables. These models were adjusted for the same covariates as the main model.

The study included several pre-planned sensitivity analyses. A substantial change in UK hypertension treatment patterns occurred in 1999, when the systolic treatment threshold in British guidelines was lowered in many patients from $160 \mathrm{~mm} \mathrm{Hg}$ to $150 \mathrm{~mm} \mathrm{Hg}$. We therefore looked for the presence of a cohort effect in our data by generating separate models for patients who had the midpoint of their treatment strategy assessment period in 1999 or earlier and those who had their midpoint in 2000 or later.
We subsequently investigated whether defining a shorter treatment strategy assessment period (three years instead of 10) would lead to misclassification of prescribing strategies. We also analyzed the effect a shorter (three years) treatment strategy assessment period would have on the calculation of the optimal systolic blood pressure target (as a large fraction of patients did not have any medication intensifications during the first three years, time to intensification and time to follow-up after intensification could not be defined). In this analysis, for patients without intensifications, we assumed that the highest blood pressure attained remained below the intensification threshold. For such patients, we therefore defined the estimated systolic intensification threshold as the highest attained non-transient blood pressure during the treatment strategy assessment period, rounded up to the nearest $10 \mathrm{~mm} \mathrm{Hg}$.

In the event that physicians would decide to alter their hypertension management strategy after a patient had an acute cardiovascular event, our approach raises a risk of bias in the presence of risk factors (for example, acute cardiovascular events) that are at the same time determined by previous exposure (for example, hypertension control) and determine subsequent exposure. ${ }^{26}$ To estimate the effect that acute cardiovascular events during the treatment strategy assessment period may have on our model, we included a variable corresponding to the presence of such an event during this period. Finally, to investigate whether time to intensification and time to follow-up have an effect on outcomes independent of frequency of visits, we introduced a variable corresponding to the total number of blood pressure measurements over the 10 year treatment strategy assessment period.

We obtained $\mathrm{P}$ values by using the type III test and used the Simes-Hochberg method to adjust significance thresholds for multiple hypothesis testing. ${ }^{27} 28$ We used SAS version 9.3 for all analyses.

\section{Results}

\section{Patients' characteristics}

We identified 149829 patients from The Health Improvement Network database with a hypertension diagnosis code and at least 10 years of subsequent primary care records between 1986 and 2010. We excluded patients who had no blood pressure measurements, were aged under 18 years, had fewer than 10 years of follow-up, had no medication intensifications during the 10 years after diagnosis of hypertension, or had missing demographic data or implausible body mass index values (fig 1). The final study population therefore consisted of 88756 adult patients (Table 1). After the treatment strategy assessment period, mean follow-up time was 37.4 months; 9985 (11.3\%) patients had an acute cardiovascular event or died.

\section{Treatment strategy and outcome risk}

In multivariable analysis, male sex, older age, obesity, diabetes, previous cardiovascular disease, chronic kidney disease, history of smoking, higher Charlson comorbidity index, and socioeconomic deprivation 


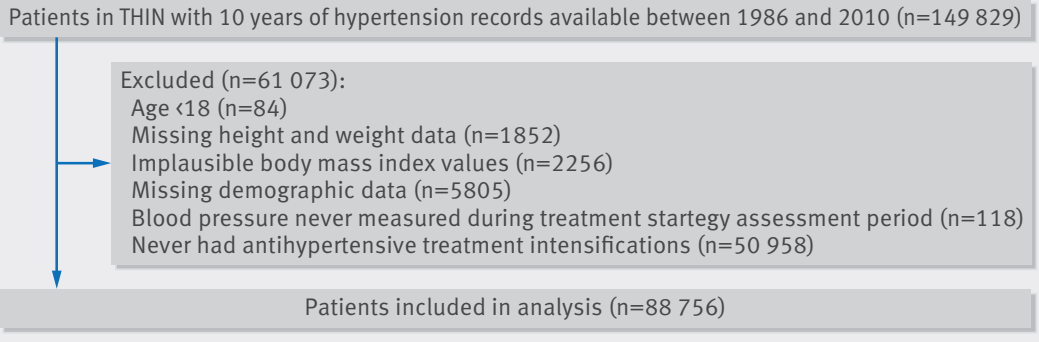

Fig 1 | Study patients and exclusion criteria. THIN = The Health Improvement Network

\begin{tabular}{|c|c|}
\hline Characteristic & Value \\
\hline No of participants & 88756 \\
\hline Mean (SD) age, years & $58.5(11.9)$ \\
\hline Male sex & $36800(41.5)$ \\
\hline Mean (SD) body mass index & $27.6(5.0)$ \\
\hline Past/current smoker & $50176(56.5)$ \\
\hline History of any cardiovascular disease & $9907(11.2)$ \\
\hline History of coronary artery disease & $6827(7.7)$ \\
\hline History of congestive heart disease & $601(0.7)$ \\
\hline History of stroke & $2450(2.8)$ \\
\hline History of peripheral vascular disease & $981(1.1)$ \\
\hline History of diabetes & $5863(6.6)$ \\
\hline Chronic kidney disease & $2420(2.7)$ \\
\hline Mean (SD) modified Charlson index & $0.27(0.6)$ \\
\hline Mean (SD) Townsend deprivation score & $2.66(1.3)$ \\
\hline
\end{tabular}

Table 2 | Effects of patients' baseline characteristics on risk of cardiovascular event or death

\begin{tabular}{|c|c|c|}
\hline Variable & Hazard ratio $(95 \% \mathrm{Cl})$ & $P$ value \\
\hline Female sex & 0.74 (0.71 to 0.77$)$ & $<0.001$ \\
\hline \multicolumn{3}{|l|}{ Age (years):* } \\
\hline$<60$ & 1.00 & - \\
\hline $60-74$ & $2.37(2.19$ to 2.57$)$ & $<0.001$ \\
\hline$\geq 75$ & 5.99 (2.54 to 6.49$)$ & $<0.001$ \\
\hline Townsend deprivation scoret & $1.09(1.08$ to 1.11$)$ & $<0.001$ \\
\hline Past or current smoker & 1.21 (1.16 to 1.27$)$ & $<0.001$ \\
\hline Modified Charlson comorbidity index $\neq$ & 1.14 (1.11 to 1.17$)$ & $<0.001$ \\
\hline \multicolumn{3}{|l|}{ Body mass index: } \\
\hline$<20$ & 1.95 (1.66 to 2.29$)$ & $<0.001$ \\
\hline $20-24.9$ & 1.00 & - \\
\hline $25-29.9$ & 0.97 (0.93 to 1.02$)$ & 0.27 \\
\hline$\geq 30$ & $1.08(1.02$ to 1.14$)$ & 0.006 \\
\hline \multicolumn{3}{|l|}{ Pre-existing medical conditions: } \\
\hline Diabetes & $1.62(1.51$ to 1.73$)$ & $<0.001$ \\
\hline Coronary artery disease & $1.48(1.40$ to 1.57$)$ & $<0.001$ \\
\hline Chronic heart failure & $1.61(1.38$ to 1.87$)$ & $<0.001$ \\
\hline Cerebrovascular disease & $1.45(1.32$ to 1.77$)$ & $<0.001$ \\
\hline Peripheral vascular disease & 1.60 (1.44 to 1.73$)$ & $<0.001$ \\
\hline Chronic kidney disease & $1.15(1.02$ to 1.30$)$ & 0.021 \\
\hline \multicolumn{3}{|c|}{$\begin{array}{l}\text { Results of multivariable Cox proportional hazards regression model of time to death from any cause or } \\
\text { cardiovascular event that included variables in tables } 2 \text { and } 3 \text {. } \\
\text { *Age categories were calculated at beginning of outcome assessment period. } \\
\text { tHazard ratio for Townsend deprivation score is per fifth increase in socioeconomic deprivation. } \\
\text { fHistory of cardiovascular disease and diabetes were omitted from calculation of modified Charlson inde } \\
\text { hazard ratio is per } 1 \text { point increase in Charlson score. }\end{array}$} \\
\hline
\end{tabular}

were associated with a greater risk of cardiovascular events or death (Table 2). Systolic blood pressure intensification thresholds of $160 \mathrm{~mm} \mathrm{Hg}$ or higher were associated with a progressively increased risk of the composite outcome (Table 3). Longer time to medication intensification was associated with a progressively greater risk of the composite outcome, beginning with the lowest fifth (0-1.4 months). Patients who had time to follow-up greater than 2.7 months also had an increased risk of the composite outcome. A sensitivity analysis that included patients with missing demographic and body mass index information gave similar results (supplementary table D).

To examine the relation between prescribing behavior and event rate without dividing systolic blood pressure intensification threshold, follow-up, and intensification time into discrete categories, we created a natural cubic spline model (fig 2). A lower systolic blood pressure intensification threshold was associated with a progressively lower risk of the composite outcome. Shorter times to medication intensification were associated with a decreased risk of cardiovascular event or death; the greatest rate of increase in risk occurred in the first nine months. A J shaped curve was noted for time to follow-up, as both very short and very long follow-up times were associated with increased risk of cardiovascular event or death.

In a secondary analysis using all cause mortality as the endpoint, we found a similar relation between systolic intensification threshold, time to intensification, time to follow-up, and all cause mortality (Table 4). Higher fifths of time to intensification were associated with progressively increased overall mortality risk, as were time to follow-up after intensification greater than 2.7 months and systolic intensification thresholds of greater than $150 \mathrm{~mm} \mathrm{Hg}$.

The sensitivity analysis for cohort effect showed no difference in the direction or significance of the associations between minimum intensification threshold, time to intensification, or time to follow-up and the composite outcome between patients with treatment strategy assessment period midpoint before compared with in or after 2000 (supplementary tables E and F). Sensitivity analysis of alternate lengths of the treatment strategy assessment period showed that with a three year treatment assessment period, $40.8 \%$ of all patients in the highest fifth for time to intensification (when we used the 10 year treatment strategy assessment period) were reclassified to lower fifths. The fraction of unintensified periods in the highest fifth that were censored also decreased with increasing length of the treatment strategy assessment period (fig 3). In the analysis of optimal systolic intensification threshold using a three year treatment strategy assessment period that included 329491 patients, intensification thresholds above $150 \mathrm{~mm} \mathrm{Hg}$ remained associated with increased risk of cardiovascular events or death. However, thresholds of $150 \mathrm{~mm} \mathrm{Hg}$ or lower were associated with progressively decreased risk, down to a minimum intensification threshold of $130 \mathrm{~mm} \mathrm{Hg}$ (supplementary table $\mathrm{G}$ ). This reflects the trend seen in the main model 
Table 3 | Effects of characteristics of treatment strategy assessment period on risk of cardiovascular event or death

\begin{tabular}{|c|c|c|c|}
\hline Characteristic & No (\%) or mean (SD) & Hazard ratio $(95 \% \mathrm{Cl})$ & $P$ value \\
\hline \multicolumn{4}{|c|}{ Minimum systolic intensification threshold $(\mathrm{mm} \mathrm{Hg})$ : } \\
\hline 130 & $12229(13.8)$ & 0.98 (0.91 to 1.07$)$ & 0.69 \\
\hline 140 & $20458(23.0)$ & 1.00 & - \\
\hline 150 & $21329(24.0)$ & $1.03(0.97$ to 1.10$)$ & 0.34 \\
\hline 160 & $17513(19.7)$ & $1.21(1.13$ to 1.30$)$ & $<0.001$ \\
\hline 170 & $8978(10.1)$ & $1.42(1.31$ to 1.55$)$ & $<0.001$ \\
\hline$\geq 80$ & $8249(9.3)$ & $1.69(1.55$ to 1.84$)$ & $<0.001$ \\
\hline \multicolumn{4}{|c|}{ Fifths of mean time to intensification (months): } \\
\hline $0-1.439$ & $17752(20.0)$ & 1.00 & - \\
\hline $1.440-4.681$ & $17751(20.0)$ & $1.12(1.05$ to 1.20$)$ & 0.009 \\
\hline $4.682-8.689$ & $17749(20.0)$ & $1.23(1.15$ to 1.32$)$ & $<0.001$ \\
\hline $8.690-15.320$ & $17753(20.0)$ & 1.19 (1.11 to 1.28$)$ & $<0.001$ \\
\hline$\geq 15.321$ & $17751(20.0)$ & 1.25 (1.17 to 1.35$)$ & $<0.001$ \\
\hline \multicolumn{4}{|c|}{ Fifths of mean time to follow-up after intensification (months) } \\
\hline $0-0.723$ & $18283(20.6)$ & 1.06 (0.99 to 1.13$)$ & 0.085 \\
\hline $0.724-1.018$ & $17524(19.7)$ & 1.00 & - \\
\hline $1.019-1.544$ & $17887(20.2)$ & $1.01(0.95$ to 1.08$)$ & 0.71 \\
\hline $1.545-2.727$ & $17537(19.8)$ & $1.07(1.00$ to 1.14$)$ & 0.050 \\
\hline$\geq 2.727$ & $17525(19.7)$ & $1.18(1.11$ to 1.25$)$ & $<0.001$ \\
\hline \multicolumn{4}{|c|}{ Mean systolic blood pressure $(\mathrm{mm} \mathrm{Hg})$ elevation over intensification threshold (\%): ${ }^{*}$} \\
\hline $1-9$ & $47173(53.1)$ & 1.00 & - \\
\hline $10-19$ & $31376(35.4)$ & $1.13(1.07$ to 1.19$)$ & $<0.001$ \\
\hline $20-29$ & $8514(9.6)$ & $1.38(1.27$ to 1.49$)$ & $<0.001$ \\
\hline $30-39$ & $1508(1.7)$ & $1.51(1.31$ to 1.73$)$ & $<0.001$ \\
\hline $40-49$ & $185(0.2)$ & $1.78(1.26$ to 2.50$)$ & 0.001 \\
\hline $\begin{array}{l}\text { Medication possession } \\
\text { ratio }\end{array}$ & $0.859(0.19)$ & 0.80 (0.73 to 0.88$)$ & $<0.001$ \\
\hline
\end{tabular}

(Table 2) and raises the possibility that more aggressive intensification thresholds have a small added benefit that our main model lacked the power to detect.

In the sensitivity analysis of the bias that might be introduced by cardiovascular events during the treatment assessment period, the presence of such an event was strongly correlated with risk of subsequent events (hazard ratio 1.92, 95\% confidence interval 1.82 to 2.02). However, the inclusion or exclusion of this variable did not alter either the direction or the significance of hazard differences between treatment strategies.

Finally, in the sensitivity analysis to determine whether the effects of time to intensification and time to follow-up are independent of the frequency of visits, the inclusion of frequency of blood pressure measurement in the model did not qualitatively change the significance or direction of the previously observed risk differences between fifths for either metric (supplementary table $\mathrm{H}$ ). Increased visit frequency was associated with increased risk of composite outcome after adjustment for time to intensification and time to follow-up (hazard ratio 1.00, 1.00 to 1.01, per visit; $\mathrm{P}<0.001$ ).

\section{Discussion}

In this large retrospective study, we examined the relation between delays in treatment of elevated blood pressure and risk of cardiovascular events or death. We found that systolic intensification thresholds higher
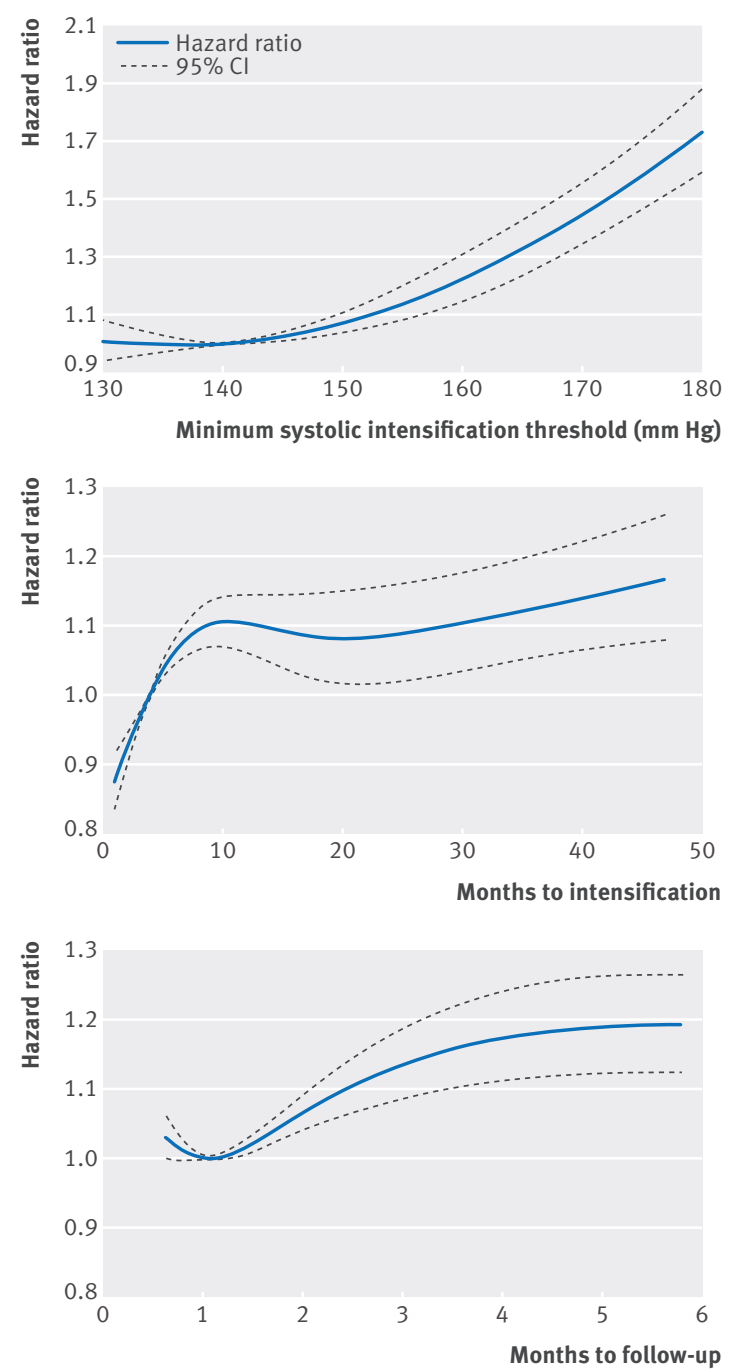

Fig 2 Effects of systolic blood pressure intensification threshold, time to antihypertensive intensification, and time to follow-up after intensification on risk of acute cardiovascular event or death. Top panel: hazard ratio for acute cardiovascular event or death in relation to systolic blood pressure intensification threshold. Middle panel: hazard ratio for acute cardiovascular event or death in relation to mean months elapsed between systolic blood pressure elevation above minimum intensification threshold and either antihypertensive medication intensification or censoring of unintensified period (via spontaneous normalization of blood pressure). Bottom panel: hazard ratio for acute cardiovascular event or death in relation to mean months elapsed between each antihypertensive medication intensification and next blood pressure measurement. Solid lines indicate hazard ratios; dashed lines indicate $95 \%$ confidence intervals calculated using natural cubic spline regression. Reference points are placed at means of respective distributions for time to intensification and time to follow-up. Knots are placed at 5th, 25th, 75th, and 95th centiles of each variable. Multivariable model was adjusted for age, sex, body mass index, smoking status, socioeconomic deprivation, history of cardiovascular disease or diabetes, other chronic medical conditions as represented by Charlson comorbidity index, minimum systolic intensification threshold, mean initial blood pressure elevation above intensification threshold, and medication possession ratio 


\begin{tabular}{|c|c|c|c|}
\hline Characteristic & No $(\%)$ or mean (SD) & Hazard ratio $(95 \% \mathrm{Cl})$ & Pvalue \\
\hline \multicolumn{4}{|c|}{ Minimum systolic intensification threshold ( $\mathrm{mm} \mathrm{Hg}$ ): } \\
\hline $130-139$ & $10853(13.4)$ & $0.99(0.90$ to 1.09$)$ & 0.80 \\
\hline $140-149$ & $18646(23.0)$ & 1.00 & - \\
\hline $150-159$ & $19724(24.3)$ & $1.05(0.97$ to 1.14$)$ & 0.22 \\
\hline $160-169$ & $16177(19.9)$ & $1.26(1.15$ to 1.37$)$ & $<0.001$ \\
\hline $170-179$ & $8253(10.2)$ & $1.42(1.28$ to 1.58$)$ & $<0.001$ \\
\hline$\geq 180$ & $7525(9.3)$ & $1.69(1.53$ to 1.87$)$ & $<0.001$ \\
\hline \multicolumn{4}{|c|}{ Fifths of mean time to intensification (months): } \\
\hline $0-1.406$ & $16233(20.0)$ & 1.00 & - \\
\hline $1.407-4.646$ & $16238(20.0)$ & $1.11(1.03$ to 1.20$)$ & 0.009 \\
\hline $4.647-8.684$ & $16236(20.0)$ & 1.24 (1.14 to 1.34$)$ & $<0.001$ \\
\hline $8.685-15.350$ & $16238(20.0)$ & $1.20(1.10$ to 1.30$)$ & $<0.001$ \\
\hline$\geq 15.351$ & $16233(20.0)$ & 1.30 (1.19 to 1.42$)$ & $<0.001$ \\
\hline \multicolumn{4}{|c|}{ Fifths of mean time to follow-up after intensification (months): } \\
\hline $0-0.723$ & $16652(20.5)$ & 1.02 (0.95 to 1.10$)$ & 0.55 \\
\hline $0.724-1.018$ & $14747(18.2)$ & 1.00 & - \\
\hline $1.019-1.544$ & $17110(21.1)$ & 1.01 (0.93 to 1.09$)$ & 0.90 \\
\hline $1.545-2.694$ & $16577(20.4)$ & 1.05 (0.98 to 1.15) & 0.18 \\
\hline$\geq 2.695$ & $16092(19.8)$ & 1.21 (1.13 to 1.30$)$ & $<0.001$ \\
\hline \multicolumn{4}{|c|}{ Mean systolic blood pressure ( $\mathrm{mm} \mathrm{Hg}$ ) elevation over intensification threshold (\%):* } \\
\hline $1-9$ & $43576(53.7)$ & 1.00 & - \\
\hline $10-19$ & $28627(35.3)$ & 1.12 (1.05 to 1.20$)$ & $<0.001$ \\
\hline $20-29$ & 7521 (9.3) & 1.31 (1.19 to 1.44$)$ & $<0.001$ \\
\hline $30-39$ & 1301 (1.6) & 1.58 (1.34 to 1.85$)$ & $<0.001$ \\
\hline $40-49$ & $153(0.2)$ & 1.98 (1.34 to 2.92$)$ & $<0.001$ \\
\hline Medication possession ratio & $0.861(0.192)$ & $0.92(0.82$ to 1.03$)$ & 0.14 \\
\hline
\end{tabular}

than $150 \mathrm{~mm} \mathrm{Hg}$ and delays of greater than 1.4 months before medication intensification after systolic blood pressure elevation above the intensification threshold were associated with an increased risk of an acute cardiovascular event or death. After each intensification of antihypertensive treatment, lack of follow-up blood pressure measurement within 2.7 months was also associated with an increased risk of the composite outcome.

\section{Comparison with other studies and current guidelines}

The systolic treatment threshold for patients with stage 1 hypertension is controversial. The guidelines of both the Eighth Joint National Committee and the European

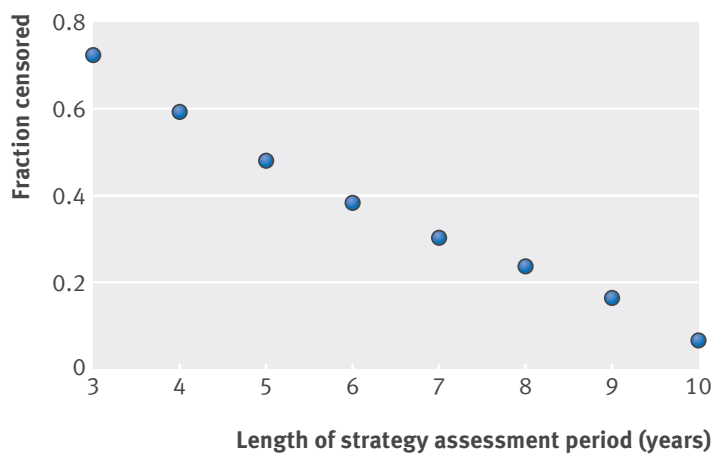

Fig 3 | Censoring of time to intensification versus length of treatment strategy assessment period (highest fifth: $>15.32$ months)
Society of Hypertension/European Society of Cardiology (ESH/ESC) suggest a target systolic blood pressure of less than $140 \mathrm{~mm} \mathrm{Hg}$ for younger patients and less than $150 \mathrm{~mm} \mathrm{Hg}$ for older patients, but they differ with regard to the age at which patients are considered older. ${ }^{8}$ On the other hand, the National Institute for Health and Care Excellence's guidelines recommend treating patients with systolic blood pressure between 140 and $160 \mathrm{~mm} \mathrm{Hg}$ only when other cardiovascular risk factors or end organ damage are present, ${ }^{6}$ and a Cochrane review found no clear evidence of benefit of medication treatment for mild hypertension without considering pre-existing diabetes or end organ damage. ${ }^{29}$ These disagreements are also reflected in guidelines issued by other major societies and reflect difficulties in weighing the available evidence in the absence of definitive data. ${ }^{7}$ No clinical trial has adequately examined the question of whether medical treatment of grade 1 systolic hypertension (140-159 mm Hg) leads to improved outcomes; the treatment of patients with systolic blood pressure between 140 and $149 \mathrm{~mm}$ $\mathrm{Hg}$ is particularly controversial. Several large randomized trials have shown cardiovascular benefit when reducing blood pressure below $140 \mathrm{~mm} \mathrm{Hg}$, but all included patients with initial blood pressure above $150 \mathrm{~mm} \mathrm{Hg} .{ }^{30-32}$ Other factors that limit the generalizability of available randomized clinical trials include the paucity of direct comparisons between blood pressure targets, incongruity of patient populations (differences in age, previous cardiovascular history, and other comorbidities), and the difficulty of achieving sufficient power to detect differences at various thresholds. ${ }^{8933} 34$ Our finding that systolic treatment targets greater than $150 \mathrm{~mm} \mathrm{Hg}$ were associated with a greater risk of acute cardiovascular event or death is therefore roughly in line with the limited data available, although other evidence suggests benefit at lower treatment thresholds in some populations. ${ }^{8} 303536$

The current ESH/ESC guidelines and previous Seventh Joint National Committee management guidelines suggest follow-up within two to four weeks or one month, respectively, after intensification of antihypertensive treatment, but these recommendations are primarily based on expert opinion rather than clinical data. ${ }^{937}$ The recent Eighth Joint National Committee guidelines do not recommend a particular interval for blood pressure assessment, but suggest intensifying treatment within a month if target blood pressure is not attained on the current treatment. ${ }^{8}$ Although direct evidence in support of these recommendations is limited, previous studies have examined the relation between frequency of visits and management of hypertension. Increased frequency of encounters has been associated with improved intermediate outcomes such as increased incidence of blood pressure control and more rapid blood pressure control..$^{38-40}$ Evidence also shows that providers often delay intensifying antihypertensive treatment when treatment goals are not met and that more frequent intensification of antihypertensive treatment leads to better blood pressure control. ${ }^{1041}$ In addition, several studies have suggested 
that delays in blood pressure control lead to increased outcome risk. The VALUE trial initially achieved greater blood pressure reductions in the amlodipine group than in the valsartan group, and a transient difference in the incidence of stroke was observed while this difference persisted..$^{42}$ In an open label extension of the Syst-Eur trial, patients who were immediately randomized to the treatment arm had a lower risk of stroke and cardiovascular complications compared with those who received delayed treatment in the extension phase. ${ }^{43}$ In this study, we show that the time to medication intensification and the time to follow-up after intensification are independent predictors of the risk of cardiovascular morbidity or death. This is, to our knowledge, the first study that has directly examined the effect of these variables on patients' outcomes. In our study population, most patients had blood pressure follow-up within 2.7 months after each medication intensification, which was the time period associated with lowest risk for the composite outcome. However, most patients did not receive medication intensification within 1.4 months. Further investigation is needed to determine whether interventions to reduce the time to medication intensification would improve outcomes.

A "J curve" corresponding to increased risk of adverse outcome at low blood pressure has previously been reported in observational studies for diastolic and occasionally systolic blood pressure, ${ }^{44-46}$ but we did not detect this phenomenon. Notably, we examined systolic blood pressure targets, whereas previous studies that noted the J curve for systolic pressure analyzed mean blood pressures without regard for treatment, which may be more vulnerable to confounding from patients' baseline illnesses. On the other hand, systolic treatment thresholds even lower than $130 \mathrm{~mm} \mathrm{Hg}$, which was the lowest treatment target we examined, may be associated with increased cardiovascular risk.

\section{Methodological considerations}

In this study, we evaluated outcomes after treatment of hypertension by defining separate time periods for the assessment of treatment strategy and outcomes. We did this to minimize the time dependent confounding caused by variations in blood pressure level, which predicts antihypertensive treatment, is itself influenced by treatment, and also affects outcome risk. A concurrent treatment strategy assessment and outcome assessment period would also introduce an undesirable bias towards systematically shorter treatment strategy assessment periods for patients who have outcomes early in the study, resulting in systematic overestimation of treatment thresholds for patients with early cardiovascular events. We chose a 10 year treatment strategy assessment period because we found that shorter treatment strategy assessment periods are vulnerable to misclassification of time to intensification, as the assessment period may end before intensification occurs. When we did alternate analyses using a three year treatment assessment period, intensification thresholds above $150 \mathrm{~mm} \mathrm{Hg}$ remained associated with increased risk. Strategies corresponding to longer time to intensification and longer follow-up times would be disproportionally affected, leading to systematic censoring bias. This study design necessarily limits our analysis to patients who had 10 years of treatment data available after the date of diagnosis of hypertension (for example, the length of the strategy assessment period). However, considering that mean life expectancy at age 65 in the United Kingdom is now more 17 years for men and 20 years for women, this may be a good initial approximation for patients in industrialized societies with routine access to medical care. ${ }^{47}$

In the natural cubic spline model, we saw a visual trend towards higher hazards when patients had very quick blood pressure follow-up, although this did not reach statistical significance in the multivariable model. This is likely to be due to confounding by indication, as we were unable to distinguish scheduled appointments from urgent care visits. Patients with a blood pressure check immediately after a previous appointment may have been seen for other urgent indications, leading to an apparent increase in outcome risk. On the other hand, delays in medication intensification longer than 10 months did not seem to be associated with a further increase in cardiovascular risk. Further investigations are needed to confirm this finding and establish its physiological basis.

This study has several additional limitations. The database we used may not have included all cardiovascular events, leading to underestimation of the cardiovascular risk associated with different antihypertensive treatment strategies. However, unless ascertainment of cardiovascular events differed between treatment strategies, this limitation should not have affected their relative cardiovascular risks that are the main findings of this study. Some of the information on medication changes could also be missing, particularly treatment adjustments made in the hospital. We did not have information on how blood pressure measurements in the database were obtained (for example, automated or manual). We assumed that providers intensify antihypertensive treatment until the target blood pressure is reached and therefore used the minimum intensification threshold as a proxy for target blood pressure. However, patients with hypertension resistant to treatment may never reach the provider's intended treatment goal, leading to potential overestimation of the systolic treatment threshold. Controversy exists surrounding the optimum systolic treatment threshold for patients with diabetes, chronic kidney disease, atherosclerosis, or advanced age, but our sample size was insufficiently large to permit stratification by these subgroups. Although we examined the effects of treatment on outcome, and our treatment strategy assessment period chronologically preceded the outcome assessment period, the retrospective nature of our data limits our ability to make causal inferences. Our study was limited to patients who were diagnosed as having hypertension by a general practitioner. However, approximately $99 \%$ of residents of the United Kingdom are registered with a general practitioner, so the 
potential for patient selection bias is less than it would be in many other countries. ${ }^{48}$

\section{Conclusion and implications}

In a population of patients with hypertension and regular access to primary care, systolic intensification thresholds higher than $150 \mathrm{~mm} \mathrm{Hg}$, delays of greater than 1.4 months before medication intensification after systolic blood pressure elevation above the intensification threshold, and delays of greater than 2.7 months before blood pressure follow-up after each antihypertensive medication intensification were associated with an increased risk of an acute cardiovascular event or death. In patients with hypertension and regular access to primary care, timely achievement of blood pressure targets and regular follow-up may be an important factor in minimizing the risk of adverse cardiovascular outcomes.

Contributors: AT, SIG, MS, and WX made substantial contributions to the study concept and design. WX and SIG did the statistical analysis. $W X$ drafted the manuscript. All authors were involved in interpretation of data and critical revision of the manuscript. AT is the guarantor.

Funding: This study was funded by the Harvard Medical School Center for Primary Care. This organization had no role in the study design; in the collection, analysis, and interpretation of data; in the writing of the report; or in the decision to submit the article for publication. All researchers acted independently of funders.

Competing interests: All authors have completed the ICMJE uniform disclosure form at www.icmje.org/coi_disclosure.pdf (available on request from the corresponding author) and declare: no financial support from any third party organization for the submitted work; no relationships with companies that might have an interest in the submitted work in the previous three years; no other relationships or activities that could appear to have influenced the submitted work.

Ethical approval: This study was approved by the Partners HealthCare System institutional review board (protocol \# 2010P002806). A waiver was obtained for the requirement of written informed consent.

Transparency: The lead author (the manuscript's guarantor) affirms that the manuscript is an honest, accurate, and transparent account of the study being reported; that no important aspects of the study have been omitted; and that any discrepancies from the study as planned (and, if relevant, registered) have been explained.

Data sharing: Full dataset and statistical code are available from the corresponding author at aturchin@partners.org. Consent was not obtained from individual participants, as the data are anonymized and the risk of identification is minimal.

This is an Open Access article distributed in accordance with the Creative Commons Attribution Non Commercial (CC BY-NC 4.0) license, which permits others to distribute, remix, adapt, build upon this work non-commercially, and license their derivative works on different terms, provided the original work is properly cited and the use is non-commercial. See: http://creativecommons.org/licenses/ by-nc/4.0/.

1 Greenland P, Knoll MD, Stamler J, Neaton JD, Dyer AR, Garside DB, et al. Major risk factors as antecedents of fatal and nonfatal coronary heart disease events. JAMA 2003;290:891-7.

2 Egan BM, Zhao Y, Axon RN. US trends in prevalence, awareness, treatment, and control of hypertension, 1988-2008. JAMA 2010;303:2043-50.

3 Lim SS, Vos T, Flaxman AD, Danaei G, Shibuya K, Adair-Rohani H, et al. A comparative risk assessment of burden of disease and injury attributable to 67 risk factors and risk factor clusters in 21 regions, 1990-2010: a systematic analysis for the Global Burden of Disease Study 2010. Lancet 2012;380:2224-60.

4 Czernichow S, Zanchetti A, Turnbull F, Barzi F, Ninomiya T, Kengne AP, et al. The effects of blood pressure reduction and of different blood pressure-lowering regimens on major cardiovascular events according to baseline blood pressure: meta-analysis of randomized trials. J Hypertens 2011:29:4-16.

5 Hsiao C], Cherry DK, Beatty PC, Rechtsteiner EA. National Ambulatory Medical Care Survey: 2007 summary. Natl Health Stat Report 2010;27:1-32.
6 National Institute for Health and Clinical Excellence. Hypertension: clinical management of primary hypertension in adults: CG127. NICE, 2011.

7 Al-Ansary LA, Tricco AC, Adi Y, Bawazeer G, Perrier L, Al-Ghonaim M, et al. A systematic review of recent clinical practice guidelines on the diagnosis, assessment and management of hypertension. PLoS One 2013;8:e53744.

8 James PA, Oparil S, Carter BL, Cushman WC, Dennison-Himmelfarb C, Handler J, et al. 2014 Evidence-based guideline for the management of high blood pressure in adults: report from the panel members appointed to the Eighth Joint National Committee (JNC 8). JAMA 2014;311:507-20.

9 Mancia G, Fagard R, Narkiewicz K, Redon J, Zanchetti A, Böhm M, et al. 2013 ESH/ESC guidelines for the management of arterial hypertension: the Task Force for the management of arterial hypertension of the European Society of Hypertension (ESH) and of the European Society of Cardiology (ESC). Eur Heart 2013;34:2159-219.

10 Okonofua EC, Simpson KN, Jesri A, Rehman SU, Durkalski VL, Egan $\mathrm{BM}$. Therapeutic inertia is an impediment to achieving the Healthy People 2010 blood pressure control goals. Hypertension 2006; $47: 345-51$

11 Egan BM, Basile JN. Controlling blood pressure in $50 \%$ of all hypertensive patients: an achievable goal in the Healthy People 2010 report? J Investig Med 2003;51:373-85.

12 Lewis JD, Schninnar R, Bilker WB, Wang X, Strom BL. Validation studies of the health improvement network (THIN) database for pharmacoepidemiology research. Pharmacoepidemiol Drug Saf 2007; 16:393-401.

13 Hall GC. Validation of death and suicide recording on the THIN UK primary care database. Pharmacoepidemiol Drug Saf 2009;18:120-31.

14 Jick H, Jick SS, Derby LE. Validation of information recorded on general practitioner based computerised data resource in the United Kingdom. BM/ 1991;302:766-8.

15 Lewis JD, Bilker WB, Weinstein RB, Strom BL. The relationship between time since registration and measured incidence rates in the general practice research database. Pharmacoepidemiol Drug Saf 2005;14:443-51.

16 Rothman KJ, Greenland S, Lash TL. Cohort studies. In: Modern epidemiology. 3rd ed. Lippincott, Williams and Wilkins, 2008.

17 Turchin A, Shubina M, Chodos A, Einbinder J, Pendergrass M. Effect of board certification on antihypertensive treatment intensification in patients with diabetes mellitus. Circulation 2008;117:623-8.

18 Levey AS, Stevens LA, Schmid CH, Zhang YL, Castro AF 3rd, Feldman $\mathrm{HI}$, et al. A new equation to estimate glomerular filtration rate. Ann Intern Med 2009:150:604-12.

19 Schulz RM, Gagnon JP. Patient behavior patterns regarding prescription refills. Contemp Pharm Pract 1982;5:150-5.

20 Khan NF, Perer R, Harper S, Rose PW. Adaptation and validation of the Charlson index for Read/OXMIS coded databases. BMC Fam Pract 2010;11:1.

21 Townsend P. Deprivation. J Soc Pol 1987;16:125-46.

22 Cox DR. Regression models and life-tables. J R Stat Soc Series B Stat Methodol 1972;34:187-220.

23 Pencina MJ, Larson MG, D’Agostino RB. Choice of time scale and its effect on significance of predictors in longitudinal studies. Stat Med 2007;26:1343-59.

24 Gail MH, Graubard B, Williamson DF, Flegal KM. Comments on 'Choice of time scale and its effect on significance of predictors in longitudinal studies'. Stat Med 2009;28:1315-7.

25 Morrison F, Shubina M, Turchin A. Encounter frequency and serum glucose level, blood pressure, and cholesterol level control in patients with diabetes mellitus. Arch Intern Med 2011:171:1542-50.

26 Robins J. A graphical approach to the identification and estimation of causal parameters in mortality studies with sustained exposure periods. / Chronic Dis 1987;40(suppl 2):139-61S.

27 Hochberg Y. A sharper Bonferroni procedure for multiple tests of significance. Biometrika 1988;75:800-2.

28 Simes RJ. An improved Bonferroni procedure for multiple tests of significance. Biometrika 1986:73:751-4.

29 Diao D, Wright JM, Cundiff DK, Gueyffier F. Pharmacotherapy for mild hypertension. Cochrane Database Syst Rev 2012;8:CD006742.

30 Zhang Y, Zhang X, Liu L, Zanchetti A. Is a systolic blood pressure target $<140 \mathrm{mmHg}$ indicated in all hypertensives? Subgroup analyses of findings from the randomized FEVER trial. Eur Heart) 2011;32:1500-8.

31 Hypertension Detection and Follow-up Program Cooperative Group. The effect of treatment on mortality in "mild" hypertension: results of the hypertension detection and follow-up program. N Engl J Med 1982;307:976-80.

32 Medical Research Council Working Party. MRC trial of treatment of mild hypertension: principal results. Br Med J (Clin Res Ed) 1985;291:97-104. 
33 Peterson ED, Gaziano JM, Greenland P. Recommendations for treating hypertension: what are the right goals and purposes? JAMA 2014;311:474-6.

34 ACCORD Study Group, Cushman WC, Evans GW, Byington RP, Goff DC Jr, Grimm RH Jr, et al. Effects of intensive bloodpressure control in type 2 diabetes mellitus. N Engl I Med 2010;362:1575-85.

35 Liu L, Zhang Y, Liu G, Li W, Zhang X, Zanchetti A, et al. The Felodipine Event Reduction (FEVER) Study: a randomized long-term placebocontrolled trial in Chinese hypertensive patients. J Hypertens 2005;23:2157-72

36 Wright JT, Fine LJ, Lackland DT, Ogedegbe G, Dennison Himmelfarb CR. Evidence supporting a systolic blood pressure goal of less than 150 $\mathrm{mm} \mathrm{Hg}$ in patients aged 60 years or older: the minority view. Ann Intern Med 2014;160:499-503.

37 Chobanian AV, Bakris GL, Black HR, Cushman WC, Green LA, Izzo JL, et al. The seventh report of the Joint National Committee on Prevention, Detection, Evaluation, and Treatment of High Blood Pressure: the JNC 7 report. Hypertension 2003;42:1206-52.

38 Lichtenstein MJ. Steele MA, Hoehn TP, Bulpitt Cl, Coles EC Visit frequency for essential hypertension: observed associations. J Fam Pract 1989;28:667-72.

39 Guthmann R, Davis N, Brown M, Elizondo J. Visit frequency and hypertension. / Clin Hypertens 2005;7:327-32.

40 Turchin A, Goldberg SI, Shubina M, Einbinder JS, Conlin PR. Encounte frequency and blood pressure in hypertensive patients with diabetes mellitus. Hypertension 2010;56:68-74.
41 Berlowitz DR, Ash AS, Hickey EC, Friedman RH, Glickman M, Kader B, et al. Inadequate management of blood pressure in a hypertensive population. N Eng / Med 1998;339:1957-63.

42 Julius S, Kjeldsen SE, Weber M, Brunner HR, Ekman S, Hansson L, et a Outcomes in hypertensive patients at high cardiovascular risk treated with regimens based on valsartan or amlodipine: the VALUE randomised trial. Lancet 2004;363:2022-31.

43 Staessen JA, Thijisq L, Fagard R, Celis H, Birkenhäger WH, Bulpitt CJ, et al. Effects of immediate versus delayed antihypertensive therapy on outcome in the Systolic Hypertension in Europe Trial. / Hypertens 2004:22:847-57.

44 Vokó Z, Bots ML, Hofman A, Koudstaal PJ, Witteman JC, Breteler MM. J-shaped relation between blood pressure and stroke in treated hypertensives. Hypertension 1999:34:1181-5.

45 Shorr RI, Somes GW. Can diastolic blood pressure be excessively lowered in the treatment of isolated systolic hypertension? J Clin Hypertens 2000;2:134-7.

46 Boutitie F, Gueyffier F, Pocock S, Fagard R, Boissel JP, for the INDANA Project Steering Committee. J-shaped relationship between blood pressure and mortality in hypertensive patients: new insights from a meta-analysis of individual-patient data. Ann Intern Med 2002;136:438-48

47 Office for National Statistics. Mortality statistics: deaths registered in England and Wales (series DR). ONS, 2012.

48 Boreham R, Airey C, Erens B, Tobin R. National surveys of NHS patients: General practice, 2002. http://www.nhssurveys.org/surveys/331

(c) BMJ Publishing Group Ltd 2015 